\title{
Future Perspectives at CERN
}

\author{
John Ellis ${ }^{1}$ \\ Theoretical Physics Division, CERN, Geneva, Switzerland
}

\begin{abstract}
Current and future experiments at CERN are reviewed,with emphasis on those relevant to astrophysics and cosmology. These include experiments related to nuclear astrophysics, matter-antimatter asymmetry, dark matter, axions, gravitational waves, cosmic rays, neutrino oscillations, inflation, neutron stars and the quark-gluon plasma. The centrepiece of CERN's future programme is the LHC, but some ideas for perspectives after the LHC are also presented.

CERN-TH/2002-119 astro-ph/0206054

Talk given at the CERN-ESA-ESO Symposium, München, April 2002
\end{abstract}

\section{Outline}

The scientific mission of CERN is to provide Europe with unique accelerators for the study of the fundamental particles of matter and the interactions between them. The scientific programme of CERN for the next decade is centred on the LHC accelerator, which is scheduled for completion in 2006, so that its experiments can start taking in 2007. A description of the LHC scientific programme is the centrepiece of this talk. The motivations for this and other new accelerators provided by ideas about possible physics beyond the Standard Model were discussed earlier at this meeting [1].

Between now and the startup of the LHC, CERN has a very limited programme of running experiments. However, scientific diversity at CERN is enhanced by a number of recognized experiments, that do not use the CERN accelerators and are not supported by CERN, but whose scientists are allowed to use other CERN facilities. In parallel with the construction of the LHC, CERN is also preparing to send a long-baseline neutrino beam to the Gran Sasso underground laboratory in Italy, in a special programme largely supported by extra contributions from interested countries. These are described before the LHC programme.

In the longer term, CERN has started thinking about its future prospects beyond the LHC. Various options have been proposed, including upgrades of the LHC, a concept for a multi-TeV electron-positron collider called CLIC, a neutrino factory, and a possible role in space experiments. These are mentioned at the end of this talk, with particular emphasis on CLIC.

At each stage in this talk, the relevances of CERN experiments to astrophysics, cosmology and space science are emphasized. The symbiotic relationships between particle physics and these subjects, that motivated this meeting, 
are amply reflected in the many connections between microphysics and macrophysics revealed in this brief survey.

\section{Present CERN Experiments}

In addition to the LHC accelerator that is currently under construction, as seen in Fig. 1, CERN has a number of lower-energy accelerators operating at energies between a few hundred $\mathrm{MeV}$ and several hundred $\mathrm{GeV}$. Protons at the lowest energies feed the ISOLDE facility, CERN's source of radio-active ions. Several experiments at ISOLDE address astrophysical issues, including a search for axions and massive neutrinos, studies of neutron-rich isotopes relevant to the supernova $r$-process, etc. [2].

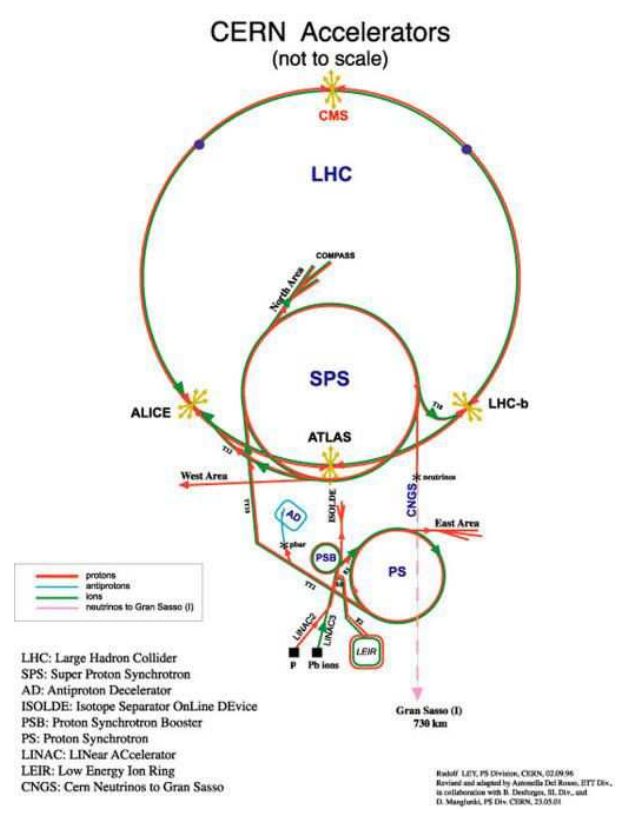

Fig. 1. Map of the CERN accelerators, showing how the LHC will be fed from the smaller SPS and PS rings via the transfer lines TI 2, TI 8. Also shown are the locations of the ALICE, ATLAS, CMS and LHCb experiments, as well as the starting-point of the $C N G S$ beam and the antoproton decelerator $(A D)$.

Protons from CERN's next-lowest-energy accelerator, the PS, are used partly to make antiprotons. These are in turn slowed down in CERN's antiproton decelerator (AD) and used to manufacture antihydrogen atoms [3] in the world's first antimatter factory [4], illustrated in Fig. 2. Their numbers are insufficient by many orders of magnitude to drive spaceships à la Star Trek, but they can be used to test matter-antimatter asymmetry in the form of CPT violation, with 
unprecedented accuracy. Scenarios for generating the baryon asymmetry of the Universe usually rely on the breaking of matter-antimatter symmetry via $\mathrm{CP}$ violation, but it has sometimes been suggested that the violation of CPT might also play a role. The AD and its associated experiments will provide some pointers on such ideas, by using laser spectroscopy to probe for differences in the energy levels of hydrogen and antihydrogen atoms.

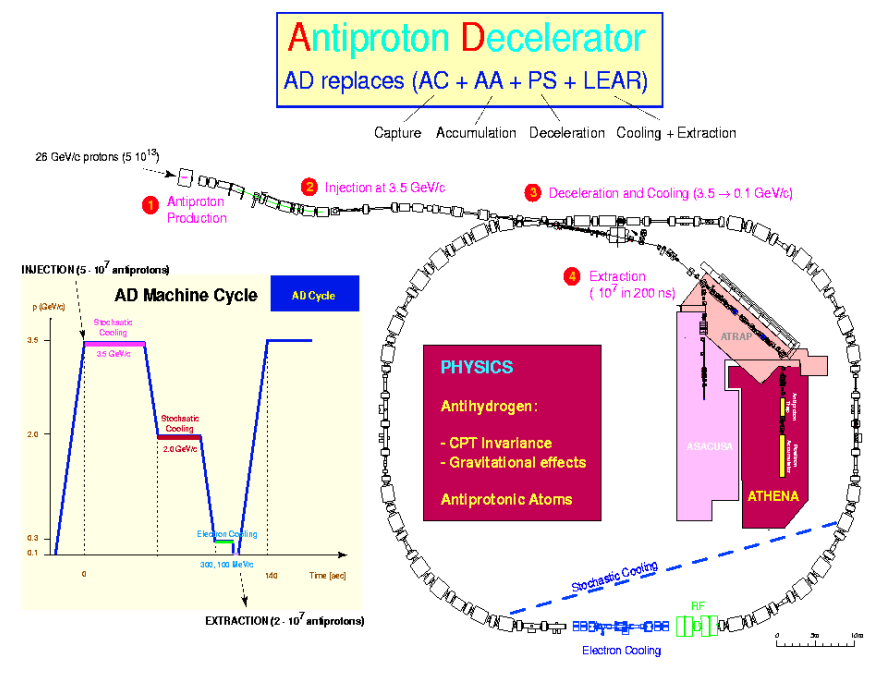

Fig. 2. Layout of the Antiproton Decelerator (AD) complex, including the three experiments ASACUSA, ATHENA and ATRAP.

CERN's current highest-energy accelerator, the SPS, is mainly used to test components for the LHC experiments, but also has a limited research programme. For example, the COMPASS experiment [5] is contributing to the understanding of the proton spin, which is relevant to calculations of the scattering of cold dark matter particles. The NA48 experiment [6] has been studying $\mathrm{CP}$ violation in kaon decays, establishing its presence directly in decay amplitudes [7], as postulated in many scenarios for baryogenesis. This an important proof of principle, but baryogenesis would require analogous direct $\mathrm{CP}$ violation the decays of different particles.

CERN is also conducting one non-accelerator experiment of interest to astrophysics, namely the CAST experiment [8] that is searching for axions from the Sun. Shown in Fig. 3, it uses a surplus superconducting LHC magnet to look for axion-to-photon conversion in a strong magnetic field, and hopes to achieve a sensitivity beyond indirect astrophysical limits on axions [9]. 


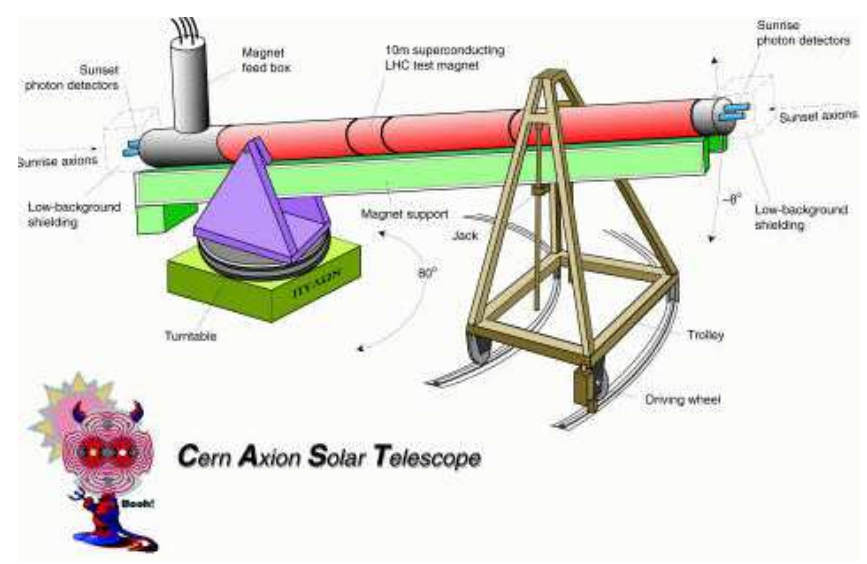

Fig. 3. Design of the CAST experiment, which will use a surplus LHC magnet to search for solar axions.

\section{Experiments Recognized at CERN}

As has already been mentioned, recognized experiments do not use CERN accelerators, and receive no financial support from CERN, but are allowed officially to use other CERN facilities, such as office space and the computer network. These concessions were originally requested by physicists who were splitting their time between some experiments that use CERN accelerators and others that do not. CERN accepts the principle of such time-sharing, and is grateful for the scientific diversity that it provides, particularly during the pre-LHC period when there are relatively few new CERN data.

\subsection{Gravitational Waves}

EXPLORER [10] was the first experiment to be recognized at CERN. It uses CERN's cryogenic facilities to keep cold its large bar detector for gravitational waves. More recently, CERN has recognized the LISA experiment [11], a trio of spacecraft that is now being designed to look for lower-frequency, longerwavelength gravitational waves in space.

\subsection{Astrophysical Antimatter}

AMS [12] was the first particle spectrometer to have been sent into space, on the space shuttle in 1998, and an improved configuration is currently being prepared to fly again for several years on the International Space Station. AMS looks, in particular, for antimatter particles such as positrons, antiprotons and antinuclei, that might be signatures of antimatter in the Universe, or of dark matter annihilations. Its first flight made interesting measurements of the Earth's cosmic-ray 
albedo [13], and set a new upper limit on anti-helium in the primary cosmicray flux [14] (see Fig. 4) that constitutes further evidence against a matterantimatter symmetric cosmology. Most of the financial support for the AMS detector comes from European funding agencies, particularly in France, Germany, Italy and Switzerland, so it is natural to have a centrally-located ground base in Europe. Two other recognized space experiments that will also look for astrophysical antimatter are CAPRICE [10] and PAMELA [15]. GLAST [16] is a satellite gamma-ray spectrometer able to look, for example, at gamma-ray bursters and/or energetic photons from the core of our galaxy that might be generated by the annihilations of dark matter particles.

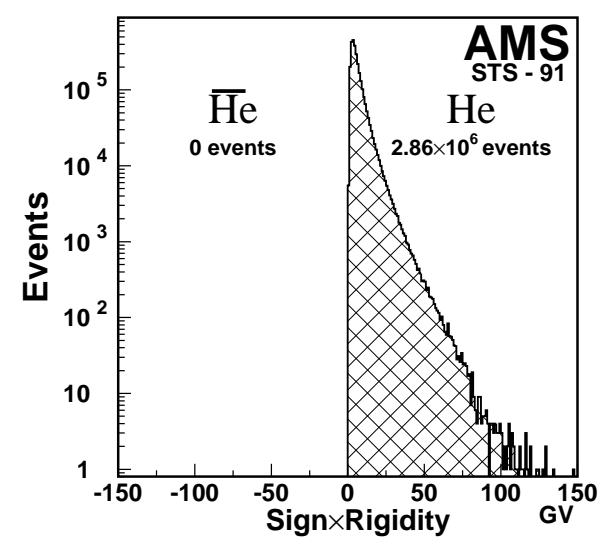

Fig. 4. The AMS experiment looked for antihelium nuclei during its first flight on the space shuttle. It found many conventional helium nuclei, but no antihelium.

\subsection{Neutrino Telescopes}

ANTARES [17] and NESTOR [18] are two underwater neutrino telescopes that will be looking for high-energy cosmic neutrinos, as might come from astrophysical sources or the annihilations of dark matter particles in the Sun and the core of the Earth.

\subsection{Cosmic Rays}

$\mathrm{L} 3+\mathrm{C}[19]$ is an extension of the L3 experiment, that made measurements with CERN's LEP accelerator, using its muon detectors and additional counters to study cosmic rays. L3+C has provided some interesting measurements of the cosmic-ray muon flux, that help constrain calculations of the atmospheric neutrino flux, and hence refine the interpretation of neutrino oscillation experiments. AUGER [20] is an ultra-high-energy cosmic-ray experiment being constructed 
in Argentina by a team from several continents, with a strong European participation. As discussed here by Watson [21], if the AGASA experiment [22] is correct, AUGER should be able to gather large numbers of events from beyond the Greisen-Zatsepin-Kuzmin cutoff [23], and tell whether they are due to compact astrophysical sources, or to the decays of very heavy metastable dark matter particles (cryptons [24]). There is currently some controversy in the energy calibrations of ultra-high-energy cosmic rays, which accelerator experiments at CERN could in principle help resolve, e.g., by a PS or SPS experiment to remeasure the spectrum of fluorescence light from nitrogen at different frequencies [21], and/or by constraining models of high-energy particle showers using data on hadron production at the LHC.

\section{Neutrino Beam from CERN to the Gran Sasso Laboratory}

CERN is currently constructing a beamline for sending neutrinos the $730 \mathrm{~km}$ to the Gran Sasso underground laboratory in Italy, called the CNGS project [25], whose starting-point is illustrated in Fig. 1. Experiments on atmospheric neutrinos suggest strongly that $\mu$ neutrinos oscillate mainly into $\tau$ neutrinos [26], but direct experimental proof is still lacking. The energy $E_{\nu} \sim 20 \mathrm{GeV}$ of the beam to be sent from CERN to the Gran Sasso is optimized for $\tau$ production via the charged-current reaction $\nu_{\tau}+N \rightarrow \tau+X$. Civil engineering was started in 2000, and the beam may be commissioned in 2006 [27].

The first experiment to be approved for the long-baseline beam was OPERA [28], which will use emulsion techniques with high spatial resolution to identify events in which $\tau$ leptons are produced. The ICARUS collaboration [29] proposes to build a 3000-tonne liquid argon calorimeter in the Gran Sasso underground laboratory, having already demonstrated the feasibility of the technique using a 600-tonne pilot module, and got approval to install it at Gran Sasso.

In the longer run, other experiments might want to use the CERN-Gran Sasso beam. For example, it has recently been proposed [30] to place a detector in the Gulf of Taranto, just off the beam axis [31], where it would be very sensitive to $\nu_{\mu} \rightarrow \nu_{e}$ transitions.

\section{LHC}

The Large Hadron Collider ( $\mathrm{LHC}$ ), under construction for installation in the $27 \mathrm{~km}$ tunnel previously used for LEP, is primarily designed to deliver protonproton collisions with a centre-of-mass energy of $14 \mathrm{TeV}$ with a luminosity of $10^{34} \mathrm{~cm}^{-} 2 \mathrm{~s}^{-1}$ [32]. It will also be able to collide lead ions at a centre-of-mass energy of $1.2 \mathrm{PeV}$ with a luminosity of $10^{27} \mathrm{~cm}^{-} 2 \mathrm{~s}^{-1}$.

The main objective of the proton-proton programme is to explore physics in a new energy range from $100 \mathrm{GeV}$ to a $\mathrm{TeV}$ and beyond. This is the energy range where the origin of particle masses is expected to be revealed, and one of the 
principal preys of the LHC will be the Higgs boson, or whatever accomplishes its task of giving masses to the elementary particles. The prospects of finding the Higgs boson are good, as seen in Fig. 5 [33]. Discovery of such an elementary scalar boson would also be interesting for cosmologists, since it is the prototype for the inflaton [34].

Most particle theorists believe that the Higgs boson will have to be accompanied by other new particles, such as those predicted by supersymmetry, which should also be accessible to the LHC, as seen in Fig. 6 [33,35]. The lightest supersymmetric particle is a leading candidate [36] for the cold dark matter thought by astrophysicists to infest the Universe.

LHC's lead-lead collisions will probe nuclear matter at temperatures and pressures typical of those in the early Big Bang when the Universe was less than about $10^{-6} \mathrm{~s}$ old, when it is thought to have taken the form of a quarkgluon plasma. Experiments at the LHC experiment will also continue studies of matter-antimatter asymmetry in decays of particles containing the bottom quark, probing whether they can be described by the Standard Model, and seeking to cast light on cosmological baryogenesis. If history is a reliable guide, the best-remembered discovery of the LHC will probably be none of these!

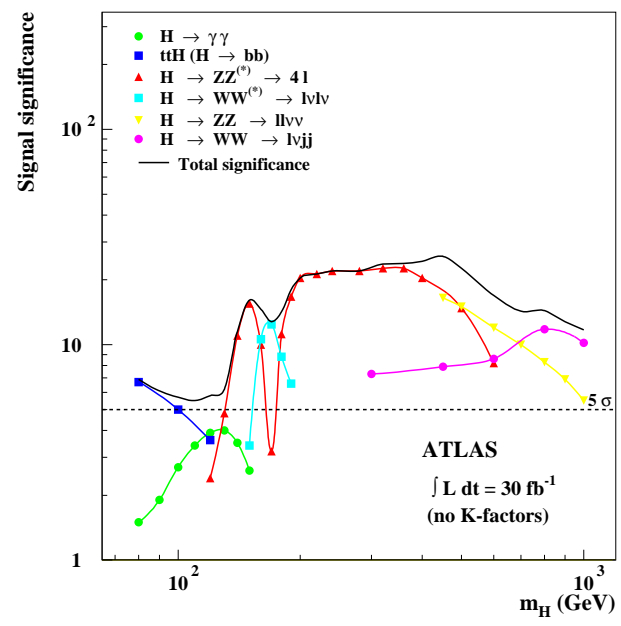

Fig. 5. Either of the major $L H C$ experiments will be able to discover the Higgs boson of the Standard Model in a variety of different decay modes, with a total significance exceeding 10 standard deviations.

The LHC experimental programme will consist of two major detectors, ATLAS [37] and CMS [38], that are designed to look for new particles and other novel phenomena at high energies, another experiment ALICE [39] directed towards studies of heavy-ion collisions, and the smaller LHCb experiment [40] looking at matter-antimatter asymmetry. New underground caverns have been dug to accommodate the ATLAS and CMS experiments, whereas ALICE and 


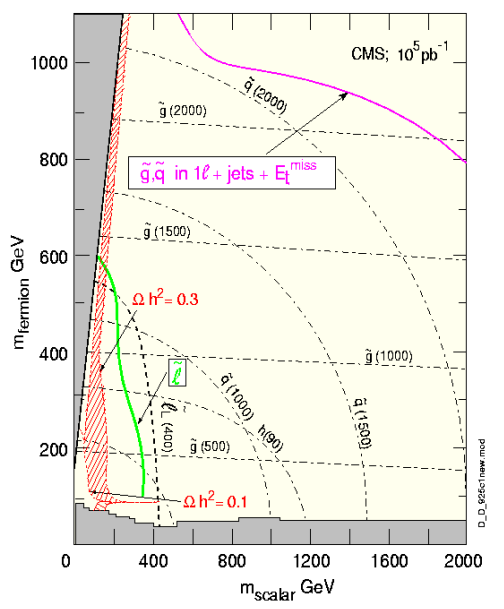

Fig. 6. Either of the major $L H C$ experiments will be able to discover supersymmetry with high significance throughout most of the parameter space where the lightest supersymmetric particle could constitute the cold dark matter, indicated by the (red) diagonal hatching.

LHC-b will be housed in caverns used previously by LEP experiments, as seen in Fig. 1. Another small experiment called TOTEM [41] will be attached to CMS to measure the total and elastic proton-proton cross sections, and a dedicated search for magnetic monopoles is also being proposed.

For the first time, the LHC accelerator is being built as a true global collaboration, with important contributions from many other laboratories besides CERN. Outside CERN's member states, important components are being provided by the United States, Russia, Japan, Canada and India. The contracts for the 1200 main-ring dipole magnets have now been placed. Pre-production models have already been delivered to CERN, and have met the specifications for accelerating protons to $7 \mathrm{TeV}$. These magnets are very challenging, since they must operate at $1.9 \mathrm{~K}$ and achieve fields of 9 Tesla. A test string of LHC magnets has been ramped successfully up to the field required to reach this design energy.

Some delays were incurred in the civil engineering for the experimental caverns and the tunnels used to transfer particles into the LHC ring, which turned out to be quite complicated. For example, the access pit to the CMS cavern had to be excavated through an underground stream, that had to be frozen with liquid nitrogen before digging was possible. The cavern itself consists of two large caves side-by-side. To support the rock above, first the wall between these caves was excavated and filled with concrete, and only subsequently could the caves themselves be dug out. The ATLAS cavern is so large that its concrete roof has to be held up by steel stays bolted into the rock above.

The civil engineering is now nearly complete, and no further delays are anticipated from this source. The principal items still on the critical path are procuring 
the superconducting cable for the main dipole magnets, which has also incurred significant delays, and the cryolines in the LHC tunnel. Because of the delays suffered so far, the scheduled completion of the LHC ring has been pushed back to the end of 2006, with first collisions in 2007.

The major detectors resemble onions, as illustrated in Fig. 7, whose concentric layers measure different types of particles produced in the collisions, such as charged particles, photons, electrons, strongly-interacting particles and muons. They include enough silicon detectors to cover a football field, (in the case of CMS) tens of thousands of lead tungstate crystals, and large superconducting magnets. The ATLAS detector is so large that its cavern could accommodate CERN's central administration building - and some wags have suggested that might be a good place to put it. Large parts of these detectors have already been constructed and delivered to CERN, ready for assembly and installation in their respective caverns. The collaborations are on course for having operational detectors ready to take useful data as soon as the LHC starts colliding protons.

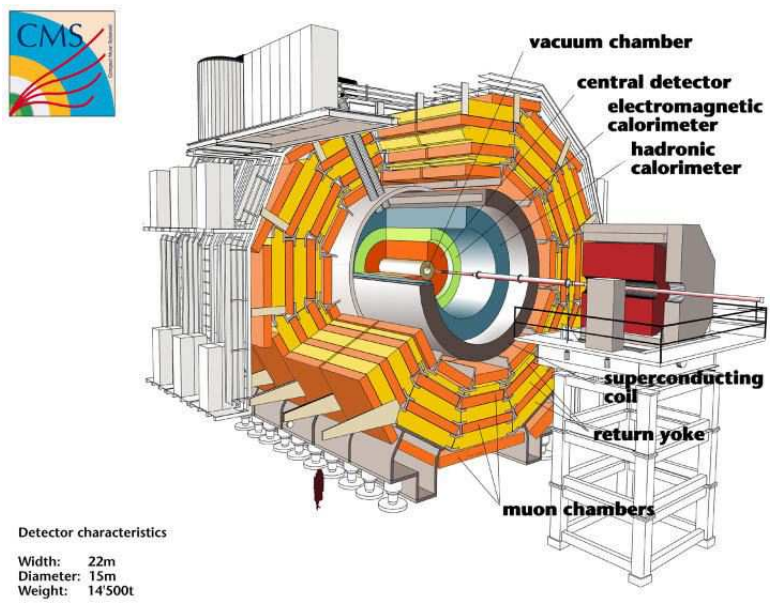

Fig. 7. Conceptual drawing of the CMS experiment, illustrating its 'onion-skin' structure, with different layers designed to detect and measure different types of particles.

The ALICE detector not only reuses an old LEP experimental cavern, it also reuses the magnet used previously for the L3 experiment. Inside it also has a large particle tracker and features specialized subdetectors for photons, electrons and muons. The putative quark-gluon plasma is not expected to have a single distinctive signature, or 'smoking gun', but rather is expected to be identified using a number of convergent indicators, such as Hanbury-BrownTwiss interferometry using particles emitted from the last-scattering surface of the expanding fireball produced by each 'Little Bang', characteristic abundances of heavier particles, and energetic photons emerging directly from inside the Little Bang, reproducing conditions early in the Big Bang, as seen in Fig. 8. 


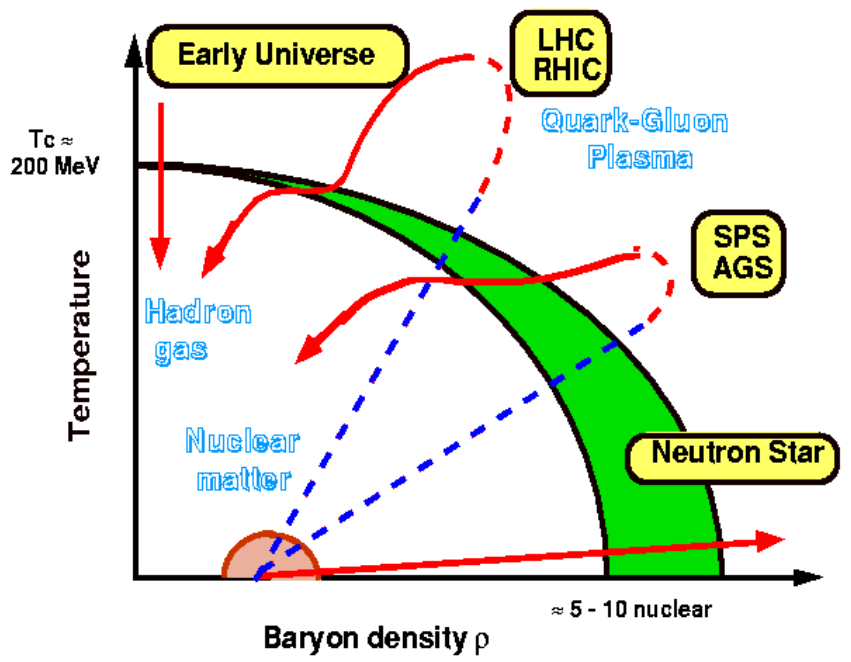

Fig. 8. Relativistic heavy-ion experiments such as ALICE at the LHC attempt to produce a hot and dense state of matter, which is thought to have been a quark-gluon plasma. The interiors of neutron stars are thought to be relatively cool but with a large baryon density, SPS and Brookhaven AGS experiments have probed higher temperatures, and the ALICE and Brookhaven RHIC experiments will probe conditions closest to those in the first microsecond of the Big Bang.

Previous experiments at CERN and BNL colliding heavy-ion beams with fixed targets provided matter under high pressures, but with relatively low temperatures and high baryon densities, reminiscent of neutron stars. More recent experiments with the RHIC heavy-ion collider at BNL have pushed to higher temperatures [42], but the LHC will come closest to reproducing conditions in the Big Bang, where the baryonic chemical potential was negligible compared to the temperature.

\section{CERN's Global Network}

The important contributions of non-European countries to the LHC accelerator have already been underlined. The signicance of their contributions to the LHC experiments is even greater: about $50 \%$ of the physicists and engineers in the experimental collaborations come from institutions outside CERN's member states, and about $30 \%$ of the total value of the components of the detectors. Each of the major LHC detectors has about 1800 participating physicists and engineers, and about fifty coutries are represented officially. As seen in Fig. 9, the total number of scientists registered as making scientific use of CERN's facilities is between 6000 and 7000, with 4000 to 5000 coming from its member states. The largest external contingents Russia and the United States, around 600 each, 
followed by Japan, Canada, Israel, Brazil, China and Korea. We collaborate with physicists from every continent except Antarctica.

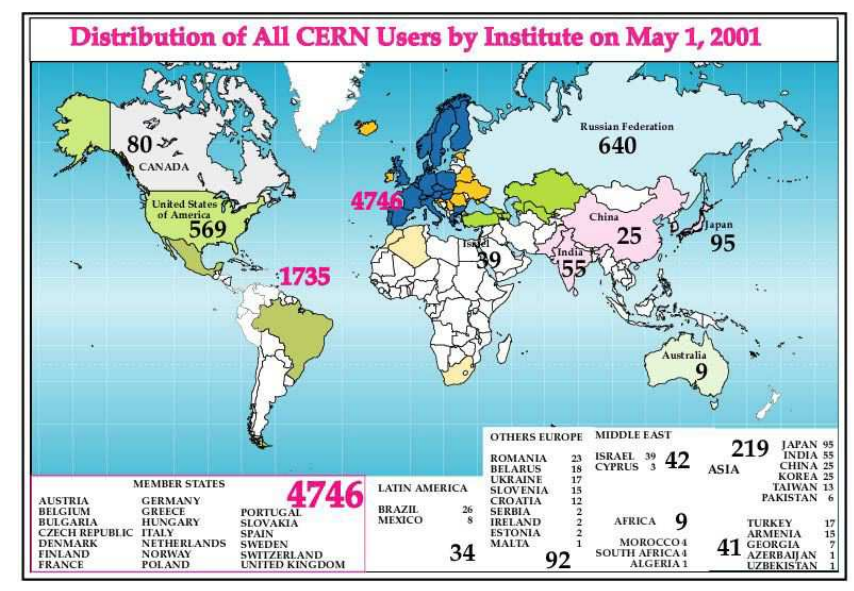

Fig. 9. High-energy physicists from around the world use CERN for their experiments. Most come from CERN's European member states, but around 2000 scientists come from elsewhere.

The age distribution of these scientific users is particularly interesting: the mode is below 30, corresponding to the large numbers of students and postdoctoral research associates that pass through CERN every year. However, more than half of these students subsequently move out of academic research, into industry, finance, etc..

\section{From the Web to the Grid}

The World-Wide Web was invented at CERN to enable all the far-flung collaborators in its LEP experimental programme to share information. Nobody at CERN anticipated the social phenomenon it would become. Now the LHC project is confronting CERN with a staggering new computing challenge.

Each of the major LHC experiments will take data at a rate of about a Petabyte $\left(10^{15}\right.$ bytes) per second. This is equivalent to about a billion people surfing the Web simultaneously, or everybody on the planet making dozens of simultaneous mobile phone calls. The great majority of these data are not interesting: perhaps only one collision in $10^{12}$ will contain a Higgs boson, for example. Therefore, the detectors' data acquisition systems are designed to discard all except one interesting candidate in every $10^{7}$ collisions, approximately. This still leaves several Petabytes of data to be recorded each year and analyzed, a task that would require 100,000 or more of today's PCs, which CERN cannot afford. 
CERN plans to tackle this problem by deploying [43] the Grid computing technology, with which the user of a desk-top or portable computer should be able to analyze data stored anywhere in the world, using CPU power wherever it is available, just as we can switch a light on without wondering where the electricity comes from. The Grid objective is transparent user access to data, programs and computing power. Applications of Grid technology to many other sciences, such as biology (e.g., the human genome project), space science and environmental science (e.g., earth observation) are also being developed, but the advent of the LHC provides a definite time-frame over which particle physicists must get a solution in place.

The amount of computing power required to simulate and analyze LHC data is set to grow much faster than Moore's Law, the rate at which the computing power of a single chip has grown historically. In the past, CERN has solved this problem by establishing farms of commodity PCs, rather than using monolithic supercomputers. The Grid project carries this decentralization to its logical conclusion, linking together farms of farms around the planet.

CERN has many collaborating partners in developing the Grid. One of the largest European projects is DataGrid, funded by the European Union and including ESA, PPARC in the United Kingdom, CNRS in France, INFN in Italy and NIKHEF in the Netherlands as well as CERN. Other European projects include CrossGrid, which involves new partners from Ireland and Central Europe, there is the DataTag project to link up with the United States, and parallel projects exist there and elsewhere. In the unique OpenLab venture, we have also attracted industrial partners to provide hardware for Grid development at CERN.

During Phase I of the LHC computing Grid project extending to 2005, the plan is to write the basic software and middleware (system management software), and to demonstrate its functioning with simulated data at a level that is a significant fraction of the eventual LHC requirement. Installation of the full LHC Grid is scheduled for the years 2006 to 2008.

\section{Options for CERN after the LHC}

Even though the LHC will only start taking data in 2007, and will continue to provide exciting data for a decade or more, the preparation times needed for new accelerator projects are so long that we at CERN have already started thinking about possible new projects after the LHC [44,45]. Several laboratories around the world have for some years already been developing plans for linear electronpositron colliders capable of centre-of-mass energies up to about $1 \mathrm{TeV}$, hoping to start one of these projects in parallel with the operation of the LHC. One of the options being considered at CERN is a possible next-generation electronpositron collider called CLIC [46], capable of higher centre-of-mass energies up to about $5 \mathrm{TeV}$ : see Fig. 10. If supersymmetry exists, this would almost guarantee accurate measurements of the properties of all the supersymmetric particles, enabling us, for example, to calculate better the density of supersymmetric relics 
from the Big Bang, and the rates at which they would scatter on conventional matter.

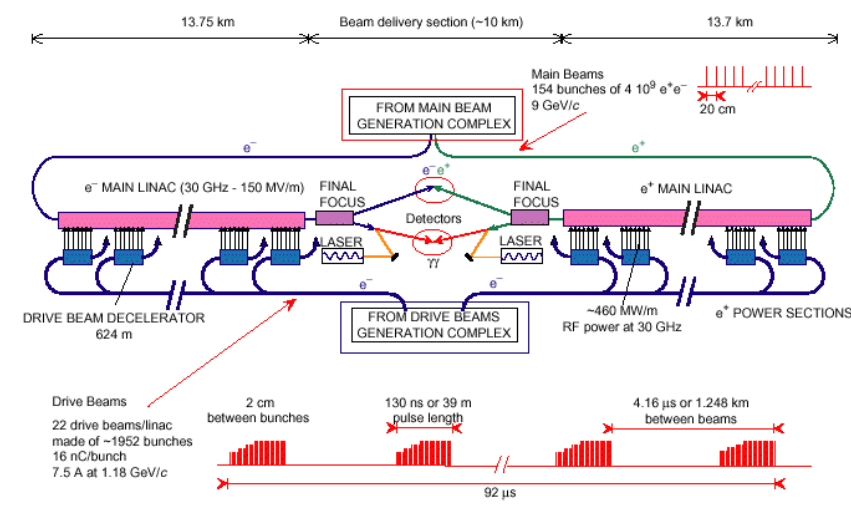

Fig. 10. Conceptual design for a linear $e^{+} e^{-}$collider capable of reaching a centre-ofmass energy of $3 \mathrm{TeV}$ or more, called CLIC, which uses high-power but low-energy drive beams to accelerate less-intense colliding beams to high energies.

In order to avoid a very long device, this energy objective would require a very high accelerating gradient. CERN proposes to achieve this by an innovative double-beam technique, in which an intense low-energy drive beam is used to generate $\mathrm{RF}$ power that is then transferred to a lower-intensity, higher-energy beam. This is the origin for the name CLIC, for 'Compact Linear Collider'. The double-beam principle has been demonstrated in a couple of test facilities, and another now under construction [47] is intended to provide an engineering demonstration of the CLIC concept. Another step towards demonstrating its feasibility might be a Higgs factory using $\gamma \gamma$ collisions generated by shining laser beams on $e^{-} e^{-}$beams colliding at around centre-of-mass energies around $150 \mathrm{GeV}[48]$.

Any new accelerator project is sure to require a global collaboration even more widely spread than the LHC. However, other projects considered at CERN have even more planetary dimensions. One possibility is a neutrino factory [49], designed to produce a controlled beam two or three orders of magnitude more intense than current long-baseline projects, using the decays of muons captured in a storage ring, as seen in Fig. 11. One of the primary objectives of such a project would be to measure matter-antimatter asymmetry in neutrino oscillations. This might require sending a neutrino beam several thousand kilometres from one continent to another, making the experiments truly global. Such experiments might cast important light on one of the favoured scenarios for baryogenesis [50], in which the decays of massive neutrinos first provide a lepton asymmetry, that Standard Model interactions subsequently convert into a baryon asymmetry. Following steps in this programme could include $\mu^{+} \mu^{-}$colliders with various 
centre-of-mass energies, possibly including one of more Higgs factories and/or a high-energy frontier machine [49].

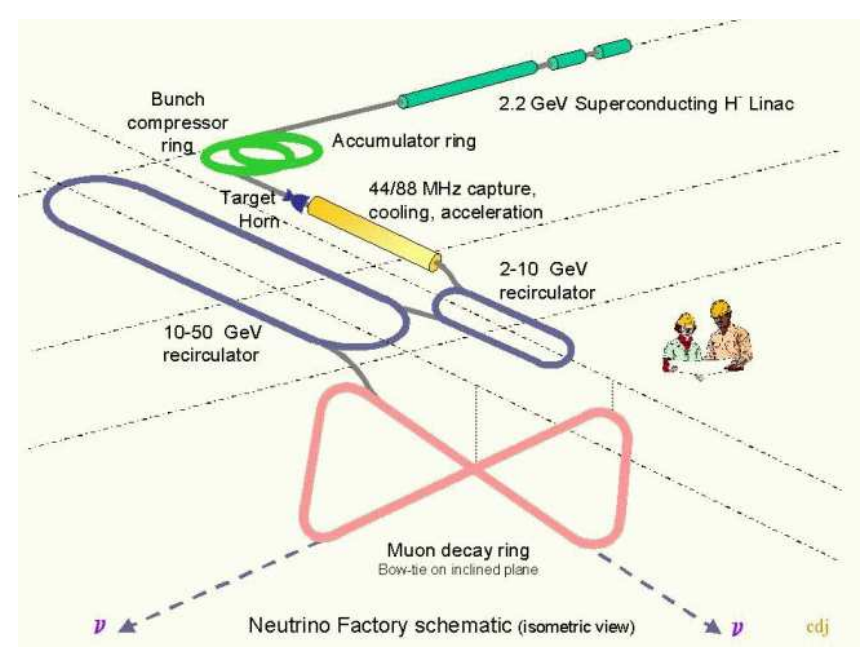

Fig. 11. Conceptual layout for a neutrino factory, based on an intense superconducting proton linac that produces many pions, whose decay muons are captured, cooled in phase space and stored in a 'bow-tie' ring. Their subsequent decays send neutrinos with known energy spectra and flavours to a combination of short- and long-baseline experiments.

Another future possibility for CERN that has attracted some interest is a more active role in space experiments. As already mentioned, CERN currently recognizes several space experiments, including AMS, CAPRICE, GLAST and PAMELA. It has a similar interest in AUGER, which is looking for ultra-highenergy cosmic rays, and EXPLORER, looking for gravitational waves. The next steps in these directions may be EUSO [51] or OWL, monitoring from space cosmic-ray impacts in even larger volumes of the atmosphere, and LISA. We at CERN certainly have legitimate scientific interests in the physics of these experiments: once the problem of mass has been sorted out, grand unification and gravity may be next on the particle physicists' agenda, and space experiments such as these might offer good ways to test them. But would CERN add significant value to such projects?

CERN will not take any irrevocable decision concerning its future before we have at least some results from the LHC, providing clearer hints where to head next. For now, CERN has its hands full constructing the LHC, with all its technical, financial, organizational, computational and physical challenges. This project provides us with us with exciting scientific perspectives for the rest of this decade and (most of) the next, providing us with plenty of time to develop one of the above ideas, or perhaps another, into a longer-term perspective worthy of CERN's mission. 


\section{References}

1. J. Ellis, talk at this meeting, see also arXiv:hep-ph/0110192.

2. For a listing of ISOLDE experiments, see: http://greybook.cern.ch/, under the ISOLDE rubric.

3. The production of antihydrogen atoms was first reported in G. Baur et al. [PS210 Collaboration], Phys. Lett. B 368, 251 (1996); Nucl. Instrum. Meth. A 391, 201 (1997); and subsequently in G. Blanford, D. C. Christian, K. Gollwitzer, M. Mandelkern, C. T. Munger, J. Schultz and G. Zioulas [E862 Collaboration], Phys. Rev. Lett. 80, 3037 (1998).

4. For a listing of $\mathrm{AD}$ experiments, see: http://greybook.cern.ch/, under the PS rubric.

5. The home page of this experiment is: http://wwwcompass.cern.ch/.

6. The home page of this experiment is: http://na48.web.cern.ch/NA48/.

7. V. Fanti et al. [NA48 Collaboration], Phys. Lett. B 465, 335 (1999) [arXiv:hepex/9909022]; A. Lai et al. [NA48 Collaboration], Eur. Phys. J. C 22, 231 (2001) [arXiv:hep-ex/0110019]; see also A. Alavi-Harati et al. [KTeV Collaboration], Phys. Rev. Lett. 83, 22 (1999) [arXiv:hep-ex/9905060].

8. The home page of this experiment is: http://axnd02.cern.ch/CAST/.

9. For a review, see: G. Raffelt, Nucl. Phys. Proc. Suppl. 77, 456 (1999) [arXiv:hep$\mathrm{ph} / 9806506]$.

10. For information about this and other experiments recognized at CERN, see: http://greybook. cern.ch/, under the 'Recognized Experiments' rubric.

11. The home page of this experiment is: http://lisa.jpl.nasa.gov/.

12. The home page of this experiment is: http://hpl3tri1.cern.ch/.

13. J. Alcaraz et al. [AMS Collaboration], Phys. Lett. B 472, 215 (2000); Phys. Lett. B 484, 10 (2000).

14. J. Alcaraz et al. [AMS Collaboration], Phys. Lett. B 461, 387 (1999).

15. The home page of this experiment is: http://wizard.roma2.infn.it/pamela/fram_des.htm.

16. The home page of this experiment is: http://www-glast.stanford.edu/.

17. The home page of this experiment is: http://antares.in2p3.fr/.

18. P. K. Grieder [NESTOR Collaboration], Nuovo Cim. 24C, 771 (2001).

19. The home page of this experiment is: http://13cosmics. cern.ch:8000/13cosmics/index.htm.

20. The home page of this experiment is: http://www .auger.org/auger.html.

21. A. Watson, talk at this meeting.

22. N. Hayashida et al., Astrophys. J. 522, 225 (1999) [arXiv:astro-ph/0008102].

23. K. Greisen, Phys. Rev. Lett. 16, 748 (1966); G. T. Zatsepin and V. A. Kuzmin, JETP Lett. 4, 78 (1966) [Pisma Zh. Eksp. Teor. Fiz. 4, 114 (1966)].

24. K. Benakli, J. R. Ellis and D. V. Nanopoulos, Phys. Rev. D 59, 047301 (1999) [arXiv:hep-ph/9803333].

25. For more information about this project, see: http://proj-cngs.web.cern.ch/proj-cngs/.

26. Y. Fukuda et al. [Super-Kamiokande Collaboration], Phys. Rev. Lett. 81, 1562 (1998) [arXiv:hep-ex/9807003].

27. The home pages of other long-baseline experiments include: K2K, http://neutrino.kek.jp/; NUMI-MINOS, http://www-numi.fnal .gov/. 
28. The home page of this experiment is: http://operaweb. web. cern. ch/operaweb/index. shtml.

29. The home page of this experiment is: http://www.aquila.infn.it/icarus/.

30. F. Dydak, private communication.

31. G. Barenboim, A. De Gouvea, M. Szleper and M. Velasco, arXiv:hep-ph/0204208.

32. For more information about this project, see: http://lhc-new-homepage.web.cern.ch/lhc-new-homepage/.

33. ATLAS Collaboration, http://atlasinfo.cern.ch/Atlas/GROUPS/PHYSICS/TDR/access.html; CMS Collaboration, http://cmsinfo.cern.ch/Welcome.html/CMSdocuments/CMSdocuments.html; F. Gianotti, talk at this meeting.

34. A. H. Guth, Phys. Rev. D 23, 347 (1981).

35. M. Battaglia et al., Eur. Phys. J. C 22, 535 (2001) [arXiv:hep-ph/0106204].

36. J. Ellis, J.S. Hagelin, D.V. Nanopoulos, K.A. Olive and M. Srednicki, Nucl. Phys. B 238 (1984) 453; see also H. Goldberg, Phys. Rev. Lett. 50 (1983) 1419.

37. The home page of this experiment is: http://atlasinfo.cern.ch/ATLAS/internal/Welcome.html.

38. The home page of this experiment is: http://cmsinfo.cern.ch/Welcome.html/.

39. The home page of this experiment is: http://alice.web.cern.ch/Alice/.

40. The home page of this experiment is: http://1hcb.web.cern. ch/lhcb/.

41. The home page of this experiment is: http://totem.web.cern.ch/Totem/.

42. For more information about this project, see: http://www.bnl.gov/RHIC/.

43. For more information about this project, see: http://lhcgrid.web.cern.ch/LHCgrid/.

44. J. R. Ellis, E. Keil and G. Rolandi, Options for future colliders at CERN, CERNEP-98-03 (1998).

45. A. De Roeck, J. R. Ellis and F. Gianotti, Physics motivations for future CERN accelerators, arXiv:hep-ex/0112004.

46. For more information about this project, see: http://ps-div.web.cern.ch/ps-div/CLIC/Welcome.html.

47. For more information about this project, see: http://ctf3.home.cern. ch/ctf3/CTFindex.htm.

48. D. Asner et al., arXiv:hep-ex/0111056.

49. For more information about this project, see: http://muonstoragerings. web.cern.ch/muonstoragerings/Welcome.html.

50. J. R. Ellis, J. Hisano, S. Lola and M. Raidal, Nucl. Phys. B 621, 208 (2002) [arXiv:hep-ph/0109125]; J. R. Ellis, J. Hisano, M. Raidal and Y. Shimizu, Phys. Lett. B 528, 86 (2002) [arXiv:hep-ph/0111324].

51. The home page of this experiment is: http://www.ifcai.pa.cnr.it/ EUSO/. 